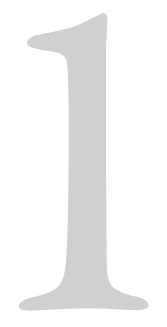

\title{
LA POLÍTICA INTERNACIONAL SUBNACIONAL DE UN MUNICIPIO INTERMEDIO: REFLEXIONES SOBRE EL CASO BAHÍA BLANCA ${ }^{1}$
}

\author{
THE ARGENTINEAN INTERMEDIATE \\ MUNICIPALITIES AND THE \\ SUBNATIONAL INTERNATIONAL \\ POLITICS: REFLECTIONS ON THE \\ BAHÍA BLANCA CASE
}

\begin{abstract}
María Sol Herrero ${ }^{2}$
Recibido: 30/04/2017

Aceptado: 10/07/2017
\end{abstract}

\section{RE S U M E N}

El artículo examina la política internacional subnacional del Municipio de Bahía Blanca (Provincia de Buenos Aires, República Argentina) en el periodo 2007-2015 indagando sobre las temáticas abordadas, los objetivos perseguidos, los medios instrumentados para alcanzarlos y los actores intervinientes. Dada la primacía de investigaciones centradas en provincias, ciudades metropolitanas y grandes aglomeraciones urbanas, el estudio se focaliza en el desempeño de una ciudad intermedia abocándose a la utilización de una metodología precisa y válida que logre reflejar las singularidades de la política internacional subnacional implementada por dichos actores.

\footnotetext{
1. Este artículo es producto de la investigación desarrollada en el marco de una Beca Doctoral de la Comisión de Investigaciones Científicas de la Provincia de Buenos Aires (Argentina) y de la participación en dos proyectos de investigación radicados en la Universidad Nacional del Centro de la Provincia de Buenos Aires (UNICEN) "Actores y dinámicas en las estrategias de desarrollo local: el rol de los gobiernos municipales en la generación de políticas de gestión internacional", financiado por el periodo 2014-2017 por la Agencia Nacional de Promoción Científica y Tecnológica de la República Argentina y "Espacios subnacionales y actores locales: dinámicas y procesos en el desarrollo de estrategias de participación internacional" acreditado durante 2015-2017 en el Programa de Incentivos a Docentes-Investigadores-Secretaría de Politicas Universitarias-Ministerio de Educación de la República Argentina. 2. Doctoranda en Ciencias Sociales (UNLU). Planta Estable en el Centro de Estudios Interdisciplinarios en Problemáticas Internacionales y Locales (CEIPIL), de la UNICEN, Tandil, Argentina. Contacto: herrerosol@gmail.com
} 
PA L A B A S C L A VE: municipios intermedios, política internacional subnacional, República Argentina, Bahía Blanca.

\section{A B S T R A C T:}

The article examines the subnational international policy of the Municipality of Bahía Blanca in the period 2007-2015. It looks at the policy's topics, its objectives, means, and stakeholders.

Given the abundance of research focused on provinces, metropolitan cities and large urban agglomerations, this study focuses on the performance of intermediate cities. The paper constructs a precise and valid methodology that reflects the singularities of the subnational international policy implemented by municipality stakeholders. Consequently, it firstly contributes by providing an analytical framework that addresses stakeholders' actions at the international level. Secondly, the paper applies this approach to the specific case of a medium-level Argentinean municipality.

K E Y W O R D S : intermediate municipalities, subnational international policy, Argentina, Bahía Blanca.

\section{I N T RO D U C C I Ó N}

A comienzos del siglo XXI, resulta indiscutible el rol protagónico que los gobiernos municipales ejercen sobre las dinámicas sociales, políticas y económicas contemporáneas. A pesar de no ser considerados como sujetos de Derecho Internacional, intervienen a través de la implementación de diversas estrategias que les permiten obtener del contexto global diversas herramientas, recursos y aliados para satisfacer sus necesidades domésticas.

Los hermanamientos, los acuerdos bilaterales, las redes internacionales de ciudades, los proyectos de cooperación, la promoción industrial, turística, cultural y comercial transfronteriza, la participación en ferias y misiones comerciales (Duchacek, 1986; Soldatos, 1990; Paquin, 2004; Batista, Jakobsen y Evangelista, 2008; Ponce Adame, 2011), constituyen nuevos escenarios de actuación que han contribuido a redefinir la relación global-local planteando nuevos desafíos a los estados subnacionales ${ }^{3}$.

3. El uso del vocablo subnacional no es unánime: existen estudios que adoptan la expresión "entidades subestatales para respetar la distinción entre estado y nación" (Romero, 2004: 48), el empleo del término subnacional es el más difundido y empleado "tanto el "actor subnacional" como el "actor subestatal" derivan de un mismo concepto integral: el "Estado-Nación" [por lo cual] su uso resulta equivalente e indistinto" (Zubelzú, 2008:36). 
Esta situación ha derivado en una profundización de los debates académicos y políticos-institucionales respecto de la conceptualización de dichas actividades, sus características y sus alcances. En Argentina, país donde se radica la investigación, de los estudios empíricos sobre la participación internacional subnacional tienen primacía aquellos focalizados a nivel provincial (Colacrai y Zubelzú, 1998; Colacrai y Zubelzú, 2004; De Marsilio, 2006) sobre los que ponen su eje en los gobiernos municipales (Dabat, 2004; CIPPEC, 2012). Dichos trabajos constatan la existencia de estrategias de inserción internacional en la generalidad de los gobiernos provinciales, de las grandes ciudades metropolitanas, así como en capitales de provincias.

Por el contrario, en este estudio se toman ciudades de menor tamaño poblacional -las intermedias- buscando ampliar el universo analítico para captar las características singulares de la inserción internacional de estos territorios. Se las define como aquellas que, en promedio, contienen entre 50.000 a 400.000 habitantes- (Vapñarski y Gorojsvsky, 1990), poseen importantes potencialidades al estar dotadas de una masa crítica institucional y empresarial más elevada que el resto de los centros urbanos, lo que les confiere un conjunto de recursos técnicos, económicos y financieros y una capacidad de innovación y de emprendimiento destacados (Michelini y Davies, 2009). De esta manera, se pretende ofrecer un aporte en un sentido, teórico -al proponerse avanzar en la generación de un marco analítico que permita dar cuenta del accionar internacional dichos actores- y empírico, -al aplicar dicho abordaje al caso concreto de un municipio de la República Argentina-.

En este marco general, se examinará la política internacional subnacional del Municipio de Bahía Blanca en el periodo 2007-2015 indagando sobre las temáticas abordadas, los objetivos perseguidos, los medios instrumentados para alcanzarlos y los actores intervinientes.

Dicha selección encuentra su justificación en una serie de características dentro del universo bonaerense que lo hacen apto como referente empírico para orientar futuros análisis comparativos de mayor complejidad: a) es un centro urbano de gran jerarquía con 301.572 habitantes (INDEC, 2010) siendo la tercera ciudad más importante del interior bonaerense luego de la capital provincial (La Plata) y del Partido de Gral. Pueyrredón; b) aporta el 2,3 $\%$ del Producto Bruto Geográfico de la Provincia de Buenos Aires; c) posee el puerto con mayor calado a nivel nacional constituyéndose en uno de los principales nodos logísticos del interior del país; d) su economía tiene un alto grado de desarrollo e impacto externo; e) es centro institucional-administrativo de la región sur bonaerense y norpatagónica, en su territorio se emplazan gran cantidad de organismos públicos y privados con una importante trayectoria internacional y f) representa un caso singular de gestión 
internacional, al ser uno de los únicos cuatro municipios intermedios del interior bonaerense en contemplar dentro de su estructura municipal un Área de Relaciones Internacionales durante el período analizado.

En cuanto a la organización del texto, en el primer apartado se presentará el marco analítico que se utilizará para el caso de estudio. En segunda instancia, se reseñará brevemente el perfil socio-demográfico, la estructura económico-productiva, infraestructura y dimensión del comercio exterior. Seguidamente, se ahondará en su estructura institucional público-privada, identificando los principales actores y organismos que guardan relación con la inserción internacional de la ciudad.

Por último, se realizará un recorrido histórico acerca de los aspectos políticos-institucionales en los que se contextualizó la política internacional subnacional para, luego, proceder al análisis de las estrategias.

\section{MARCO ANALITICO PARA EL CASO DE ESTUDIO: DIMENSIONES DEANÁLISISY A S P ECTOS M ETODOLÓG ICOS}

En el presente apartado se presentarán los lineamientos teóricos y metodológicos que guiarán el análisis.

Partiendo de considerar que la participación externa de las entidades subnacionales se encuentra materializada a través de la «política internacional subnacional», se la define como la decisión política y la herramienta pública de los gobiernos locales que se ocupa de impulsar la inserción internacional a través de una estrategia tendiente a aprovechar, articuladamente, las necesidades del territorio con las oportunidades del contexto exterior (Calvento, 2016).

En Argentina, tales contactos se producen a partir de una relación complementaria y colaborativa con el Estado nacional (Russell, 2010; Colacrai, 2013), que posibilita compartir parte del núcleo blando de la política exterior (Salomón, 2007). En este marco, los gobiernos locales no fijan el contenido de la política exterior -es decir el "qué", dado que esa es un área reservada al gobierno nacional- pero tienen posibilidades de influir en el "cómo" (Colacrai y Zubelzú, 2004).

En base a los análisis pre-existentes y teniendo en cuenta trabajos previos (Calvento, Lorenzo y Herrero, 2014) se han definido dos dimensiones para describir y sistematizar la participación internacional de las ciudades in- 
termedias: las estrategias de relacionamiento institucional internacional y las políticas de posicionamiento internacional. Las mismas incluyen las estrategias reseñadas por Borja y Castells, 1997; Romero, 2004; Vigevani, 2006; Salomón, 2007; Batista, Jakobsen, y Evangelista, 2008, entre otros, y retoman elementos de las clasificaciones establecidas por García Segura (1996), teniendo en cuenta que contemplan estrategias de presencia directa e indirecta, principalmente de carácter formal, desarrolladas en el ámbito transregional y global, que implican políticas de cooperación. En ese sentido, las estrategias de relacionamiento institucional internacional agrupan a los vínculos formales, directos e indirectos, que se establecen con la finalidad de generar, especialmente, vías de cooperación política y técnica en el ámbito transregional y global, operacionalizándose a través de dos subdimensiones: relaciones de carácter bilateral y multilateral.

Las relaciones bilaterales se materializan a través de acuerdos entre ciudades o entre instituciones internacionales para establecer vínculos que incluyan el intercambio de experiencias relativas a cuestiones de la realidad local, así como la búsqueda de soluciones a los problemas comunes. Dentro de este tipo de relaciones, la estrategia más utilizada es la de los hermanamientos de ciudades, aunque también pueden registrarse otros tipos de acuerdos bilaterales como actas, y convenios de cooperación.

Respecto a las relaciones multilaterales, estas se conforman a través de redes u organizaciones de ciudades, permitiendo una inserción más dinámica que amplía el intercambio de información y experiencias. En las relaciones multilaterales distinguimos entre las transregionales y globales, considerando en las primeras a la Red de Mercociudades, al Foro Consultivo de Ciudades y Regiones del Mercosur (FCCR) y a los Comités de Integración, entre otras iniciativas; en tanto que, en las segundas la Red de Cooperación Internacional Descentralizada URBAL es la más destacada.

En cuanto a las herramientas de posicionamiento internacional, son aquellas de carácter unilateral desarrolladas con el objetivo de promocionar y posicionar en el exterior a los actores subnacionales. Entre ellas se diferencian las políticas locales de comercio exterior, como las Rondas de Negocios y Misiones Comerciales; de las políticas que promueven el establecimiento de una imagen territorial, como las políticas de Marca-Ciudad. En ese sentido, las políticas municipales de comercio exterior fomentan las actividades de exportación a través del asesoramiento, brindando información y capacitación sobre el comercio internacional a los interesados; y de la intermediación, estructurando redes de contactos internacionales a través de la conformación de Rondas de Negocios y Misiones Comerciales y la parti- 
cipación en Ferias Internacionales. Por su parte, el marketing de ciudades es una política pública, creada y coordinada por el gobierno subnacional, destinada a potenciar las capacidades de los territorios, desplegar sus ventajas competitivas y posicionarlos nacional e internacionalmente.

En relación a estas dimensiones adoptadas para describir y analizar la participación internacional del municipio de Bahía Blanca debe dejarse explicitado que las mismas no serán analizadas como compartimentos estancos, sino como variables que sólo pueden entenderse en el marco de la realidad político-institucional, económico-productiva y socio-demográfica del estado subnacional seleccionado como referente empírico del trabajo.

FIGURA $\mathbf{N}^{\circ} 1$

DIMENSIONES DE POLÍTICA INTERNACIONAL SUBNACIONAL

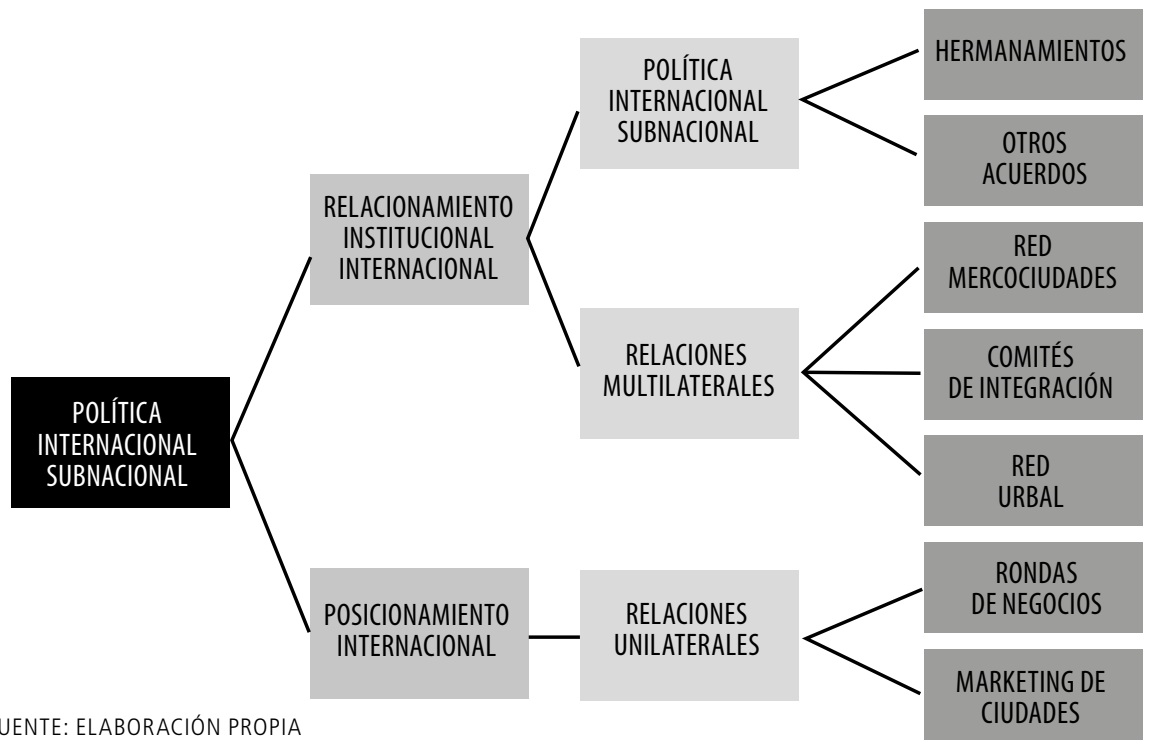

En cuanto a la estrategia metodológica, ésta se sustentó en un estudio de caso a través de un diseño de investigación mixto con triangulación de métodos (Forni, 2009), de aplicación independiente y resultados complementarios (Todd y Nerlich, 2004).

El enfoque de la investigación contempló el análisis de fuentes primarias, principalmente de diversos documentos oficiales e institucionales (Reyes y Hernández, 2008) emitidos por organismos oficiales. Esta fundamentación documental aparece como testimonio de información original a partir de la 
evidencia directa de personas u organismos involucrados en el tema-objeto de estudio dado que por considerar que cualquier política pública genera un sinnúmero de documentos escritos que es necesario reconocer, seleccionar y examinar (Müller, 2006). Se utilizaron documentos e informes de gestión emitidos por distintos organismos gubernamentales, normativa municipal, ordenanzas, decretos, gacetillas de prensa, boletines oficinales, información publicada en medios gráficos y digitales del ámbito local, entre otros.

Dicha compilación y análisis se complementó con la realización de entrevistas semiestructuradas ${ }^{4}$ a actores centrales del municipio seleccionado. Además de obtener la voz de aquellos sobre los cuales no se contaba con posicionamientos documentados, éstos sirvieron para jerarquizar la masa de información recolectada sobre los varios tipos de documentos -normativos, institucionales, de gestión, comunicación, etc.- interpretarla y aportarle un orden y coherencia (Müller, 2006).

De esta manera, se apeló entonces a una "triangulación en la recolección de datos" con el objetivo de contrastar un determinado conjunto de observaciones con otros (Forni, 2009) utilizando complementariamente las técnicas de recolección interactivas (entrevistas exploratorias) y no interactivas (análisis documental) (Reyes y Hernández, 2008).

El análisis e interpretación de los datos no fue un proceso disociado de la recolección sino que ambos se desarrollaron de manera simultánea y complementaria (Hernández Sampieri; Fernández y Baptista, 2006).

En cuanto a los alcances de la investigación, en lugar de la generalización, adopta relevancia el criterio de "transferencia" o "traslado" (Maxwell, 1992; Hernández Sampieri, Fernández y Baptista 2006), que hace referencia a la posibilidad de que los resultados puedan aplicarse a otros contextos similares, entendiendo este proceso no como una transferencia directa, sino brindando pautas generales para futuros estudios sobre el mismo problema, y la posibilidad de visualizar reflexiones similares en otros ambientes.

4. Dichas entrevistas fueron realizadas durante en el año 2015 recabando los testimonios de Guillermina Inchausti (Encargada de la Agencia de Desarrollo), Silvia Cordinalesi (Directora de Ceremonial del Municipio), Rafael Puelma Claro (Cónsul de Chile en Bahía Blanca), Elisa Quartucci (Concejal) y Laura Benedetti (Subsecretaria de Relaciones Internacionales de la Universidad Nacional del Sur). 
En Argentina, el sostenido proceso de urbanización ha acrecentado la participación relativa de las aglomeraciones de tipo intermedio, que han ido adquiriendo una posición destacada: de los 2.164 municipios de Argentina, aproximadamente 109 pertenecen a la categoría de municipios intermedios. En conjunto, contienen cerca de 15.000.000 de habitantes (INDEC, 2010), el $40 \%$ de la población del país.

La mayor cantidad de municipios intermedios se encuentran concentrados en cuatro provincias: Santa Fe, Córdoba, Mendoza y Buenos Aires, las cuales registran más de 5 localidades de ese tamaño. El caso de la provincia de Buenos Aires resulta distintivo, ya que contiene a 40 municipios de tales características, es decir, el $43 \%$ del total de municipios intermedios argentinos.

Dentro de esta caracterización se encuentra el Municipio de Bahía Blanca conformado por la ciudad homónima, cabecera del partido, y las localidades de Ingeniero White, General Daniel Cerri y Cabildo. Abarca una superficie de $2.247 \mathrm{~km}^{2}$ y está ubicado geográficamente a $647 \mathrm{~km}$ de la Ciudad Autónoma de Buenos Aires, en el sudoeste de la provincia, limitando con los partidos de Villarino, Tornquist, Coronel Pringles y Coronel Rosales.

Según el último Censo Nacional de Población, Hogares y Vivienda de 2010, registra un total de 301.572 habitantes (INDEC, 2010), convirtiéndose en el decimoséptimo puesto a nivel nacional y la tercera ciudad más importante del interior bonaerense luego de la capital provincial (La Plata).

Herrero (2014) define al distrito bahiense como uno de los principales nodos logísticos del interior del país: su conexión con el resto del país esta facilitada por rutas nacionales y provinciales, vías férreas y aéreas. La vinculación de economías regionales del sudeste bonaerense y el norte patagónico con los mercados internacionales se realiza a través del complejo portuario -conformado por Puerto Rosales, Puerto Ingeniero White y Puerto Galván- el único del país que permite operar con buques de gran calado por su profundidad de 50 pies.

Desdeuna perspectiva económica, constituyeunimportantecentroindustrial y de servicios dotado de una estructura productiva amplia y diversificada, lo cual se evidencia en los últimos datos disponibles sobre la desagregación 
MAPA $\mathbf{N}^{\circ} \mathbf{1}$

UBICACIÓN GEOGRÁFICA MUNICIPIO DE BAHÍA BLANCA, PROVINCIA DE BUENOS AIRES, REPÚBLICA ARGENTINA

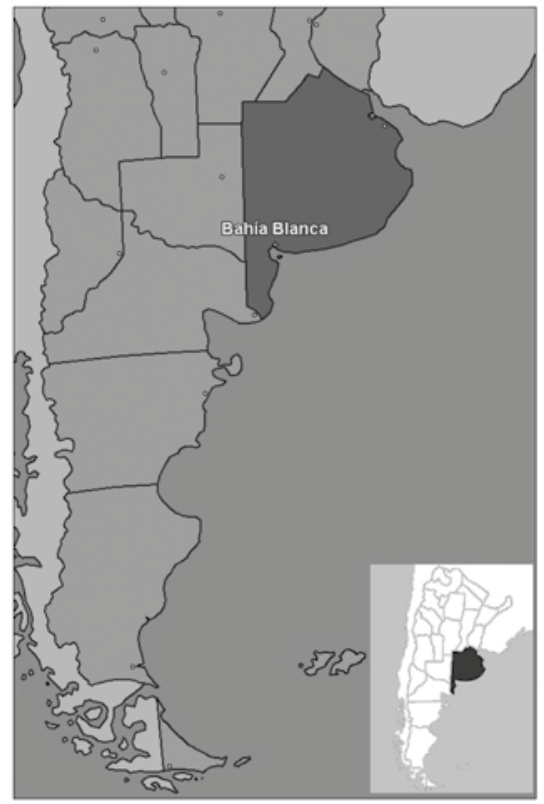

FUENTE: EDICIÓN PROPIA EN BASE A IMÁGENES EXTRAÍDAS DE WWW.FILKHBR.COM

\section{GRÁFICO $\mathbf{N}^{\circ} 1$}

ESTRUCTURA RELATIVA DEL PBg de BAhía BLANCA. AÑo 2003

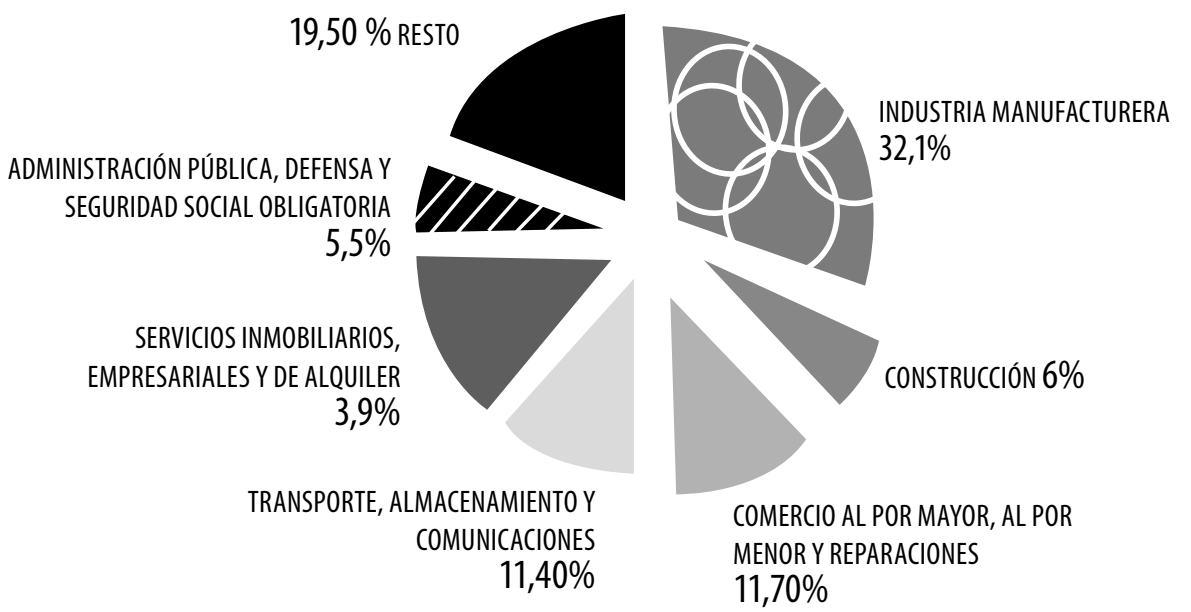

FUENTE: ELABORACIÓN PROPIA EN BASE A LOS DATOS DISPONIBLES EN DIRECCIÓN PROVINCIAL DE ESTADÍSTICA DE LA PROVINCIA DE BUENOS AIRES. 
municipal del Producto Bruto Geográfico de la Provincia de Buenos Aires. Realizando un análisis desagregado de dichos datos se observa, en primer lugar, que el Producto Bruto Geográfico (PBG) municipal para el año 2003 era del 2,3\% del total provincial, dato no menor si se tiene en cuenta que el PBG se concentra en los partidos del conurbano bonaerense (Dirección Provincial de Estadística de la Provincia de Buenos Aires, 2003) ${ }^{5}$.

Debido a las características de su puerto, Bahía Blanca ocupa un lugar relevante en la participación de las exportaciones nacionales: desde hace una década, su participación es de un $10 \%$ en toneladas exportadas y de un $6 \%$ en el monto $\mathrm{FOB}^{6}$, lo cual significa que cada 100 toneladas que exporta el país, 10 salen por Bahía Blanca, ocupando el tercer puesto del ranking de aduanas con mayor volumen de exportaciones, y el quinto en monto de operaciones anuales (CREEBBA, 2015). Brasil y China han sido los principales destinatarios del 55\% de las toneladas exportadas, destacando productos tales como trigo, petroquímicos, polímeros de etileno, urea, polietilenos y policloruro de vinilo (PVC), malta, cebada, combustibles como el propano y el gas licuado de petróleo (GLP), soja, aceites vegetales de soja y girasol, polímeros de etileno y pesquería (CREEBBA, 2015).

De lo anterior se desprende que el perfil exportador local está asociado con las cadenas productivas agroindustrial, petroquímica y de combustibles. Dentro de los veinte principales productos exportados, más de la mitad reciben algún grado de transformación dentro de Bahía Blanca, lo cual impacta favorablemente en el entramado productivo local (Herrero, 2016).

Ahora bien, esta diversificada estructura productiva se materializa en la presencia de gravitantes cámaras empresariales con asiento en la ciudad, entre otras: la Unión Industrial Bahía Blanca (UIBB), la Asamblea de Pequeños y Medianos Empresarios (APYME); la Corporación del Comercio, Industria y Servicios de Bahía Blanca, el Instituto de Desarrollo Empresario Bonaerense (IDEB), la Bolsa de Cereales, Oleaginosos, Frutos y Productos de Bahía Blanca, la Bolsa de Comercio de Bahía Blanca, el Consorcio de Gestión del Puerto de Bahía Blanca, (Herrero, 2016).

5. Al 2017, no se reportan mediciones actualizadas de dicho indicador.

6. Cláusula de comercio internacional, que se utiliza para operaciones de compraventa en que el transporte de la mercancia se realiza por barco. Acrónimo del término en inglés Free On Board, "franco a bordo, puerto de carga convenido". (www.aduanaargentina.com) 
Adicionalmente, dentro del complejo entramado institucional se destacan dos universidades nacionales de gestión pública: la Universidad Nacional del Sur (UNS) y la Universidad Tecnológica Nacional-Facultad Regional Bahía Blanca (UTN-FRBB), y también la sede administrativa de la Universidad Provincial del Sudoeste (UPSO). La interacción de dichas instituciones con el sector productivo local y regional, vinculado al mercado interno y al comercio internacional, es múltiple y abarca variadas dimensiones cuyo análisis excede el presente trabajo.

Por otro lado, la presencia de consulados y de organizaciones de migrantes en su territorio, representan una consecuencia directa de la importancia histórica y contemporánea de los procesos migratorios en este distrito: es asiento de 7 oficinas consulares (Brasil, Chile, Dinamarca, España, Francia, Italia y Noruega), y cuenta con más de una veintena de organizaciones y asociaciones de extranjeros europeos y latinoamericanos, que favorecen sin duda la red de vinculaciones y proyección internacional del municipio (Nicolao, 2015) ${ }^{7}$.

En suma, estos elementos permiten afirmar que la ciudad cumple con la caracterización de Michelini y Davies (2009) respecto de las aglomeraciones intermedias: constituye un polo de desarrollo económico, centro comercial, financiero e institucional que dinamiza el interior bonaerense y, más aún, extiende su influencia al centro y sur argentino. Además, desempeña un rol relevante a escala nacional, dada su importante infraestructura portuaria, vial y ferroviaria, su diversidad de sectores productivos, sus características geográficas y la alta calificación de sus recursos humanos, que permiten su proyección como actor subnacional al ámbito internacional. Dichas particularidades están acompañadas por la diversidad y cantidad del entramado público- privado local, que evidencian la dimensión de su masa crítica institucional y empresarial que le otorga especial particularidad como centro institucional-administrativo de la región sur bonaerense y norpatagónica, que, además, constituye uno de los pilares sobre los que se edificó la política internacional el Municipio de Bahía Blanca.

7. Como rasgo singular, la ciudad cabecera constituye una tradicional receptora de migraciones internacionales, contando al 2010 con un total de 13.502 extranjeros, que representa el 4,48\% de su población total, superando sensiblemente la media del interior bonaerense (3,8\%). Más del $70 \%$ de esta población es originaria de países limítrofes, siendo la comunidad chilena la más importante dentro de ese grupo, pero también residen en el partido migrantes de distintos paises europeos y asiáticos (INDEC, 2010). 


\section{L A P OLÍTICA I N T E R N A C I O NA L \\ $S$ U B N A C I O NAL D E L M U N I C I P I O D E B A H I A B A NCA : ASPECTOS POLÍTICOS- I NS T I T U C I O N L E S}

\subsection{ASPECTOS INSTITUCIONALES}

Para analizar el rol internacional asumido por el Municipio de Bahía Blanca durante el período 2007-2015, es necesario realizar algunas consideraciones que involucran décadas previas.

Durante la gestión del Agrimensor Jaime Linares, intendente durante tres períodos consecutivos del 1991 al 2003, en el marco de la puesta en marcha del Plan Estratégico de Bahía Blanca del año 1999, se impulsó el "Programa de identidad y posicionamiento de Bahía Blanca", el cual tuvo como objetivo incrementar la acción de la ciudad en Redes de Ciudades buscando la promoción de las oportunidades a través de políticas de intercambio y cooperación entre municipios en temáticas relacionadas con la formación y mejoramiento de los recursos humanos, fomento de nuevas actividades productivas para ampliar la base económica; promoción y posicionamiento de la ciudad tanto a nivel nacional como internacional.

Para cumplimentar estos propósitos, se formularon los proyectos: "Consolidación de Bahía Blanca en las redes de Mercociudades y URB-AL" y el "Plan de Marketing de Ciudad", cuyos lineamientos principales serán abordados posteriormente.

A partir del año 2007, con la asunción del Dr. Cristian Breitenstein ${ }^{8}$, apareció un gran interés en potenciar el rol internacional a través de la diversificación de las estrategias de vinculación internacional y la redinamización de actividades informales de mandatos anteriores. Esto respondió, en primer lugar, a la existencia de una trayectoria anterior, a los cambios en el modelo político y económico argentino, favorable para ciudades de tamaño intermedio como Bahía Blanca, pero también a la propia concepción del nuevo funcionario, al concebirlas como herramientas necesarias para la

8. Anteriormente a su asunción cumplió funciones como Concejal de Bahía Blanca por dos períodos legislativos consecutivos: años 1999-2003 y 2003-2007. También fue Asesor del Consejo de la Magistratura de la Nación, de la Cámara de Diputados de la Provincia de Buenos Aires y cumplió similar función en la de Diputados de la Nación. De profesión Abogado, recibido en la Universidad de La Plata, luego obtuvo el título de Licenciado en Filosofía, otorgado por la UNS, donde también se desempeñó como docente. Esta incursión en ámbitos locales, provinciales y nacionales, como asi también su paso por la docencia universitaria, fueron experiencias cruciales que lo llevaron a generar una serie de cambios en su gestión como Intendente, tanto en la visión sobre el rol que debía cumplir Bahía Blanca, como así también en la necesidad de nuevas (Herrero, 2014). 
gestión municipal (Herrero, 2016).

En consonancia con esta nueva percepción, en su primer discurso, aseguró que crearía dentro de la propia estructura municipal una Agencia de Relaciones Internacionales, "para vincularnos con el mundo e ir a buscar lo que nos ofrece, mostrando el potencial de toda la región" (Breitenstein, 2007).

Esta iniciativa se materializó en el Decreto 110 N $^{0} 1.808$ del año 2008, que reglamentó la creación de una dependencia gubernamental específica bajo jurisdicción directa del intendente ${ }^{9}$. La misma permitiría que las iniciativas de vinculación internacional adquirieran carácter institucional regular con el objetivo de: "maximizar la inserción de Bahía Blanca en el mundo otorgándole una adecuada visibilidad dentro del marco global, desarrollando estrategias en coordinación con los tres niveles de gobierno, y el conjunto de actores públicos y privados cuyas dinámicas contribuyan a la conformación de una plataforma común para la internacionalización de Bahía Blanca entre los cuales se encuentran Universidades, organizaciones no gubernamentales, colectividades extranjeras, Consulados y agencias consulares, instituciones de difusión de culturas extranjeras, entidades de promoción económica, entre otros" (www.bahiablanca.gov.ar).

En un inicio, esta Agencia dependió directamente de la intendencia y posteriormente, por modificaciones en el organigrama, pasó a funcionar en la órbita de la Secretaría de Gobierno. No obstante, el carácter de «Agencia» le imprimió cierta transversalidad en su operatoria.

En esta etapa, según Di Meglio y Nicolao (2016), los recursos de planificación disponibles provinieron, principalmente, de agencias provinciales, nacionales e internacionales vinculadas directa o indirectamente a la temática. En términos de infraestructura, tuvo edificio propio, lo cual representó un símbolo de cierta independencia en sus funciones; igualmente se le asignó presupuesto, aunque los recursos establecidos eran escasos, y contaba con personal altamente capacitado para desempeñar funciones en la misma.

Adicionalmente, se establecieron vinculaciones con otras dependencias creadas durante el mismo período como, por ejemplo, la Agencia de Desarrollo (creada por ordenanza No 14.640, en 2008), la Agencia Municipal de Ciencia y Tecnología y el Consorcio de Promoción y Desarrollo de las Actividades Turísticas (creado por ordenanza No 14.494 en 2007).

Durante 2007-2011, las definiciones de actuación internacional bahiense es-

9. Al conformarse como agencia supondría una posición transversal en el organigrama. Desde el año 2010, por una cuestión administrativa interna paso a depender de esa secretaria sin por eso modificar el vínculo con el intendente. 
tuvieron determinadas positivamente por la creación de la Agencia que, aunque presentó una posición no jerárquica en el organigrama, su rol fue valorizado por los funcionarios públicos ${ }^{10}$ y principalmente estimulado por el intendente municipal. Fue destacable también el involucramiento de recursos humanos capacitados en temáticas pertinentes y el acceso a sus cargos por vías formales, y por último, las articulaciones al interior del gobierno definiendo un estilo de administración descentralizado, y la disponibilidad de recursos de información, infraestructura y financieros (Calvento, 2012).

Ahora bien, en 2011, ante la solicitud de licencia de Breitenstein para ocupar el cargo de Ministro de Producción, Ciencia y Tecnología de la Provincia de Buenos Aires, asumió como intendente, el Dr. Gustavo José Bevilacqua ${ }^{11}$ quien por entonces ocupaba el cargo de primer concejal electo.

Durante su mandato (2011-2015) se disolvió la Agencia de Relaciones Internacionales, y los temas que manejaba esta área pasaron la órbita de la Dirección de Ceremonial del Municipio, dependiente de la Secretaría Privada de la Intendencia, la cual articula directamente con Turismo, Deportes y Cultura (Quartucci, 2013). Asimismo, se evidenció un interés menor del intendente en funciones sobre la temática específica, aspecto asociado, en muchos casos, a la dificultad de mostrar resultados materializables de corto plazo de las vinculaciones ganando en prioridad los asuntos de agenda local tradicionales (Consultora Pontis, 2012)

Teniendo en cuenta lo anteriormente señalado, en los subsiguientes apartados se caracterizarán las dimensiones de política internacional subnacional del Municipio.

10. Entrevistas realizadas a Silvia Corinaldesi (Directora de Ceremonial Municipio de Bahía Blanca, 11/5/2015) y Elisa Quartucci (Concejal, 16/06/2015).

11. Abogado, recibido en la Universidad de Buenos Aires, cursó la carrera de Especialización en Contratación y Documentación Notarial en la Universidad Notarial Argentina, ejerció la docencia universitaria en la carrera de Derecho de la Universidad FASTA (sede Bariloche), y siguió estudios de postgrado (Especialización en Derecho en Alta Tecnología de la Universidad Católica Argentina y Maestría en Derecho Administrativo de la Universidad Austral). En forma paralela a su actividad profesional, desarrolló actividades empresariales (Di Meglio y Nicolao, 2016). 


\subsubsection{LAS ESTRATEGIAS DE RELACIONAMIENTO INSTITUCIONAL INTERNACIONAL}

A) Las Relaciones Bilaterales: Hermanamientos de ciudades y otros acuerdos de cooperación

En el caso de Bahía Blanca, su experiencia en este tipo de estrategias de relacionamientos se remonta al año 1967 en ocasión del hermanamiento con Jacksonville (Estados Unidos), retomándose en la década del 80' con Fermo (Italia) y manteniendo una actividad continua en los 90' y 2000, con los gobiernos subnacionales de Talcahuano, Reus, Ashdod, Cienfuegos y Dalian. Según datos del Informe de Gestión 2008-2009 de la Agencia de Relaciones Internacionales, Bahia Blanca cuenta con ocho ciudades hermanas: Jacksonville (Estados Unidos), Talcahuano (Chile), Reus (España), Fermo (Italia), Cienfuegos (Cuba), Ashdod (Israel), Piura (Perú) y Dalian (China). Por su parte, Christchurch (Nueva Zelanda), Santos (Brasil) y Saint Nazaire (Francia) que habían sido declaradas ciudades amigas, continuaban al 2015 en tentativas de hermanamiento.

Respecto a las motivaciones del relacionamiento, priman las similitudes en términos de infraestructura: Talcahuano, Cienfuegos, Ashdod y Dalian son ciudades portuarias. En cuanto a Fermo, la relación responde a vínculos históricos, dado que Bahía Blanca concentra el mayor número de inmigrantes procedentes de esa región (Herrero, 2014). Por su parte, los vínculos preexistentes con Talcahuano, Dailan y Reus han sido antecedentes para el desarrollo de iniciativas que impactaron en la estructura económica-productiva y profundizaron los contactos comerciales, como se desarrollará más adelante.

Durante el período 2011-2015 no se han firmado nuevos hermanamientos, aunque la presencia de consulados -Chile, Italia, Brasil, España- y asociaciones de inmigrantes cooperan mediante sus demandas e iniciativas para profundizar los vínculos existentes y generar nuevos, tal es el caso de la ciudad de Santos (Brasil) en tratativas de hermanamiento.

En cuanto a los acuerdos bilaterales, se destaca la iniciativa de cooperación técnica "La nueva dimensión urbana y logística de las ciudades" firmada en el marco del Convenio de Colaboración Franco-Argentino con GIE-ADEFRANCE, organismo financiado por el Ministerio de Economía de Francia. La misma tuvo como objetivo identificar las obras de infraestructuras para mejorar la competitividad y se desarrolló conjuntamente con investigadores y personal técnico de la UNS y el Consorcio de Gestión del Puerto. 
Asociado a esto, se destaca el acuerdo de cooperación firmado entre el puerto de Bahía Blanca y el puerto francés de Nantes-Saint Nazaire, para incrementar los intercambios entre dichos puertos, contribuyendo, a la evolución de las relaciones comerciales entre la Unión Europea y MERCOSUR. En relación al período 2011-2015 no se registraron nuevos acuerdos de ninguna índole.

\section{B) Relaciones Multilaterales: Red Mercociudades, Comités de Integra- ción y Cooperación Descentralizada a través de redes (URB-AL)}

\section{Red Mercociudades}

Bahía Blanca forma parte de la Red Mercociudades desde la IV Cumbre realizada en Montevideo en Septiembre de 1998, durante la intendencia de Jaime Linares. Sin embargo, su mayor activismo durante el período analizado se observa a partir del 2009 al integrar diferentes Unidades Temáticas: Desarrollo Económico Local, Desarrollo Social, Educación, Turismo y Planificación Estratégica, Cultura, Desarrollo Urbano y Cooperación Descentralizada, siendo designada como sub-coordinadora en las últimas dos. A principios de 2012, durante la intendencia interina del Dr. Gustavo Bevilacqua, se mantuvo la participación en la Unidad Temática de Ciencia, Tecnología y Capacitación.

Su participación ha resultado sumamente enriquecedora porque, además de la realización de talleres e intercambio de experiencias en la gestión pública se generó un espacio para el desarrollo de políticas de comercio exterior, como las rondas de negocios o los vínculos para establecer misiones comerciales ${ }^{12}$.

\section{Comités de Integración ${ }^{13}$}

Los Comités de Integración son instancias de participación pública y privada, creadas a partir de la apertura democrática en Chile y Argentina, para promover la integración mediante el tratamiento bilateral de temas de interés.

Desde el 2010, durante la gestión Breitenstein, forma parte del Comité de Integración de la Región de los Lagos, compuesto por las provincias de Neuquén, Río Negro, Chubut, la ciudad de Bahía Blanca y las cuatro regiones colindantes de Chile: Bío Bío, Araucanía, Los Ríos y Los Lagos. Esta decisión significó un paso hacia la integración de la región norpatagónica

12. Entrevista realizada en 16/06/2015 a Guillermina Inchausti, de la Agencia de Desarrollo de Bahía Blanca. 13. Reconocen su antecedente en el Tratado de Paz y Amistad firmado entre ambos paises en 1984. A partir de este momento según Silva Soura y Morán León (2010) comienza la formalización de las relaciones subnacionales con la creación de los Comités de Frontera luego denominados Comité de Integración. 
a partir de la conexión de centros de producción con terminales portuarias, abriendo nuevas alternativas logísticas para los flujos de comercio exterior. La participación en estos procesos refuerza sus vínculos con regiones chilenas y evidencia el interés de estas en estrechar relaciones comerciales, posibilitando así la unión de las economías en línea con el caminoo emprendido por el MERCOSUR.

En este marco, es destacada la importante colaboración con el Consulado de Chile en Bahía Blanca, materializado en la realización de numerosas actividades en conjunto ${ }^{14}$.

Esta participación tiene estrecha relación con los Corredores Bioceánicos ejes de integración de carácter subnacional "puentes terrestres" que unen puntos distantes y facilitan la conexión con otros mercados extra regionales (Colacrai, 2010).

El Corredor Bioceánico Trasandino del Sur es un proyecto que pretende unir las ciudades de Bahía Blanca y Talcahuano para ofrecer a la Argentina un acceso al Océano Pacífico y a Chile al Atlántico. Si bien se realizaron diferentes gestiones para la concreción del proyecto, durante el período analizado no se ha podido formalizar, por la magnitud, diversidad de los actores involucrados y temas gravitantes que incluye.

En marzo de 2012, durante el mandato de Bevilacqua, tuvo lugar la $1^{\circ}$ Jornada de Representantes Gubernamentales del Corredor Bioceánico Trasandino del Sur-Ruta Lógica con el objetivo de reactivar la idea del corredor bioceánico. La misma se realizó por iniciativa de los intendentes de las ciudades de Bahía Blanca y de Zapala (Neuquén), en el marco de la Feria de la Producción, el Trabajo, el Comercio y los Servicios del Sur Argentino (FISA) ${ }^{15}$.

Asimismo, en 2013, Bahía Blanca fue sede del $31^{\circ}$ Encuentro del Comité de los Lagos que tuvo como principal tema a tratar la difusión de la plataforma logística regional. En conjunto, estas iniciativas refuerzan las ideas respecto del rol protagónico de la ciudad como polo logístico y de negocios aunque denotan que deben profundizarse los consensos y coordinación política para ver materializados resultados concretos.

14. Entrevista realizada en 16/06/2015 al Cónsul de Chile en Bahía Blanca, Rafael Puelma Claro (Periodo en funciones: 10/2/2014-2016).

15.La Feria de la Producción, el Trabajo, el Comercio y los Servicios del Sur Argentino (FISA) es la exposición anual multisectorial más importante del sur argentino. Tiene como principal objetivo fomentar la vinculación entre los distintos actores de la producción, la industria, el comercio, centros de estudios, trabajadores, organismos gubernamentales y no gubernamentales y consolidarse como un espacio de encuentro y herramienta de impulso y desarrollo para las ciudades que conforman el Sudoeste de la Provincia de Buenos Aires y las provincias de La Pampa, Río Negro, Neuquén y Chubut (www.fisa.com.ar). 


\section{Cooperación Descentralizada a través de redes (URB-AL)}

En cuanto a la cooperación internacional descentralizada a través de redes se destaca el Programa URB-AL en el cual Bahía Blanca ejerció la coordinación del proyecto "Impacto del crecimiento del sector industrial químico y petroquímico en la gestión urbana de ciudades portuarias". Asimismo, ha participado de "Las ciudades como promotoras de intercambios entre PyMEs $^{16}$ de América Latina (AL) y la Unión Europea (UE)" coordinado por la Diputación Provincial de Huelva (España) y “Gestión y control de la urbanización" coordinado por la Municipalidad de Rosario (Argentina).

El primero tenía como objetivo de mejorar las capacidades de gestión del desarrollo urbano en los gobiernos locales de ciudades portuarias, el segundo buscaba establecer vínculos de cooperación comerciales, entre pequeños y medianos empresarios de ciudades de AL y la UE y el tercero, planteaba articular el desarrollo urbano en términos económicos.

Más allá sus divergencias, poseen como denominador común la preocupación por aportar desde estas instancias al desarrollo de la ciudad: mejorar la infraestructura y el entramado productivo-comercial, potenciar la condición de Ciudad-Puerto, incrementar la relevancia del complejo químico y petroquímico y las PyMEs en una ciudad intermedia como Bahía Blanca (Municipalidad de Bahía Blanca, 2009).

En los años recientes, las transformaciones establecidas en la tercera fase del Programa URB-AL afectaron su metodología, pasando de una articulación en Redes a la generación de consorcios lo cual limitó la posibilidad de participación de los municipios pequeños y medianos lo cual repercutió en una merma en dichas actividades.

\subsubsection{POLÍTICAS DE POSICIONAMIENTO INTERNACIONAL}




\section{A) Comercio exterior}

Durante el período analizado se registran numerosos vínculos para generar rondas comerciales al exterior. Se destaca la presencia de una oficina de la Fundación Export.Ar ${ }^{17}$ en el edificio de la Unión Industrial de Bahía Blanca que fomenta las misiones comerciales de empresarios locales y regionales.

Dichas iniciativas se materializan en políticas concretas, como el asesoramiento y la asistencia para la promoción del comercio exterior, la integración regional y la cooperación internacional. En este sentido, se destaca el Área de Comercio Exterior de la Agencia de Desarrollo del Municipio: dicha oficina tuvo tres líneas de trabajo centrales en el este período con las cuales colaboró -mientras funcionó- la Agencia de Relaciones Internacionales y que promocionó individualmente cuando esta se desintegró: capacitaciones; promoción en ferias y misiones comerciales y asesoramiento técnico. Sin embargo, las acciones de comercio exterior se fueron paralizando especialmente en 2015, siendo cada vez menor el número de PyMEs que participan en comercio exterior ${ }^{18}$. A través de sus actividades, ha articulado con el Consorcio de Gestión del Puerto, con sectores gremiales empresariales y con el Centro de Despachantes de Aduana, con la Subsecretaría de Relaciones Económicas Internacionales de la Provincia de Buenos Aires ${ }^{19}$, y Fundación Export.Ar.

En cuanto a las políticas concretas de intermediación en comercio exterior, se retoma como ejemplo la Misión Comercial e Institucional a Talcahuano organizada en 2010 conjuntamente con la Agencia de Relaciones Internacionales y la Agencia de Comercio Exterior, que refleja una política internacional articulada, donde los antecedentes provinieron tanto de relaciones bilaterales o multilaterales.

Asimismo, en el marco del Plan de Acción 2011 del “Programa Integrado

17. La Fundación Export.Ar es una institución mixta integrada por el sector público y privado, que trabaja en coordinación con la Cancillería. Fue creada en el año 1995 con el propósito de "asistir a la comunidad empresarial en sus esfuerzos por comercializar con eficacia sus productos competitivos en el plano internacional, con miras a acceder, ampliar y diversificar sus exportaciones" (www.exportar.org.ar/). Cuenta con una Sede Central y 63 ventanillas distribuidas en todo el pais; 21 de ellas se sitúan en la Provincia de Buenos Aires.

18. La crisis económica global que se desata en 2008 y sus repercusiones en la economía nacional en los años subsiguientes provocaron dos efectos negativos: una restricción de los fondos de cooperación disponibles, que se acentuó debido a que Argentina pasó ser calificado como pais de renta media; y grandes dificultades para exportar, en especial por parte de las PyMEs, lo que acarreo desinterés del empresariado local por las políticas de promoción ofrecidas por los municipios.

19. Es un área dependiente del Ministerio de Producción, Ciencia y Tecnología que tiene como objetivo generar una mayor y mejor inserción de la producción provincial en los mercados internacionales y posicionar a la provincia como destino de proyectos de inversión que favorezcan la generación de empleo y la innovación tecnológica (http://www.mp.gba.gov.ar) 
de Promoción Comercial, Inversiones y Desarrollo de Mercados Externos"20 se llevó a cabo una Misión Comercial Multisectorial a Brasil organizada por la Subsecretaría de Comercio Internacional del Ministerio de Relaciones Exteriores y Culto, la Fundación Export.Ar y la Agencia de Desarrollo como entidad local.

En tal sentido, se buscaron identificar los productos y sectores que ofrecerían buenas perspectivas para su comercialización en el principal socio estratégico de la Argentina y miembro del MERCOSUR. Los sectores alimenticios, componentes eléctricos; plásticos y sus manufacturas, sector químico, software y servicios informáticos -de gran peso en la estructura económica-productiva bahiense- se mostraron como el principal interés en el mercado brasileño. En el año 2012 se concretó una misión multisectorial a Santiago de Chile, en la cual se mantuvieron 70 reuniones bilaterales en el marco de una misión comercial conformada por 12 empresas de la provincia. Cabe recordar la relevancia de Chile como el tercer destino en importancia de las exportaciones de la Provincia de Buenos Aires y uno de los principales destinos de la carga del Puerto de Ingeniero White.

Durante el período de Bevilacqua se continuó con la participación en la Feria FISA y se complementó, en 2014 y 2015, con la realización de la Ronda de Negocios Internacional de la Industria Electrónica, impulsada por el Departamento de Jóvenes Empresarios de la Unión Industrial de Bahía Blanca, con el objetivo de establecer un espacio de encuentro entre empresas, emprendedores e instituciones de investigación y desarrollo para potenciar oportunidades de negocios en un sector estratégico como la Tecnología.

Asimismo, se intensificaron la relaciones a nivel protocolar con la ciudad de Dailan (China), como producto de esta vinculación funcionarios municipales acompañados, en algunos casos, por empresarios y representantes del sector productivo con el objetivo de fortalecer el intercambio comercial.

En conclusión, se afirma que la participación en Mercociudades y los hermanamientos ha fomentado la generación de políticas locales de comercio exterior. La característica distintiva durante el período tuvo que ver con la articulación de dichas iniciativas con las organizadas y financiadas por organismos provinciales y nacionales de promoción de las exportaciones como el Ministerio de Relaciones Exteriores y Culto, la Fundación Export. Ar y a nivel provincial por el Ministerio de Producción, Ciencia y Tecnolo- 
gía a través de su Subsecretaría de Relaciones Económicas Internacionales.

En efecto, en el plano local se mantuvo activa la relación tanto con instituciones del gobierno nacional, provincial así como otros vínculos con diferentes embajadas.

\section{B) Marca ciudad}

En relación a este tipo de políticas, en Bahía Blanca se ha trabajado en el desarrollo de una imagen ciudad con el apoyo de diferentes organizaciones que, por medio de acuerdos con el gobierno municipal, convinieron en lanzar una nueva imagen que refuerce atributos de identidad y referencia (Municipalidad de Bahía Blanca, 2011).

El gobierno de Cristian Breitenstein se propuso el desarrollo de una política que permitiera a la ciudad destacar en el mapa nacional e internacional reforzando su rol de capital regional desde el punto de vista productivo, turístico, educativo y cultural (Municipalidad de Bahía Blanca, 2009); para lograrlo, se utilizaron dos herramientas: la confección de un libro digital con información sobre la ciudad y el desarrollo del Programa "Bahienses en el Mundo".

El citado libro, se presenta como una estrategia de compilación de las características principales de la ciudad con el objetivo de atraer inversiones seleccionando estratégicamente sectores a promocionar: el puerto atlántico, la conexión bioceánica con Talcahuano, la zona franca Bahía Blanca-Coronel Rosales, el polo petroquímico, el parque industrial y los recursos humanos técnicos-científicos.

Por su parte, el Programa Bahienses por el Mundo buscó "coordinar, facilitar, promover y apoyar iniciativas orientadas a lograr una efectiva vinculación de los bahienses en el mundo como socios estratégicos para el desarrollo de Bahía Blanca" (www.bahiablanca.gov.ar). A través de este programa se realizan encuentros anuales con los bahienses por el mundo permitiendo al gobierno municipal estar presente en diversos países: España, Italia, Brasil, Chile, Holanda, Francia, México, China, Perú, Alemania, Israel, entre otros.

El proceso de construcción de la marca tuvo un hito durante el 2011, cuando se desarrolló el seminario “Desarrollo estratégico de la Marca de Bahía Blanca", evento en el cual participaron funcionarios, delegados municipales, consejeros escolares, representantes de la Universidad Nacional del Sur y distintas entidades representativas de la ciudad.

Durante el 2011-2015 no se observan nuevas iniciativas al respecto ni un 
dinamismo de las existentes.

\section{CONSIDERACIONES FINALES}

El artículo buscó analizar la política internacional subnacional del Municipio de Bahía Blanca durante el período 2007-2015. A priori se estableció que la creación de la Agencia de Relaciones Internacionales resultaba un caso de particular interés en el universo de municipios intermedios bonaerenses por diversos motivos: la ubicación estratégica de Bahía Blanca como ciudad-puerto vinculada al comercio internacional, su amplio hinterland en el que confluye la producción de una vasta región, y una estructura productiva diversificada y con fuerte peso del sector industrial; además, por la existencia previa de organismos públicos, sectores no gubernamentales y privados que conforman una densa y articulada trama institucional, y con una importante trayectoria preexistente en vinculación internacional. En este contexto, la Agencia de Relaciones Internacionales apareció como la materialización de un estilo de gestión municipal que intentó diversificar las oportunidades del contexto internacional dotando de organización dichas actividades.

Si bien se mantuvo la actividad exterior, esta tuvo altibajos en su intensidad: durante la gestión encabezada por Cristian Breitenstein fue crucial su concepción acerca de la importancia del posicionamiento internacional de la ciudad y la creación de la Agencia, como una herramienta necesaria y estratégica para la gestión municipal.

Esta gestión, que articuló e institucionalizó las actividades pre-existentes, las dotó de una mayor dinámica, alentando a que se desarrollaran un abanico de estrategias singulares en el marco de las acciones de municipios de tamaño intermedio argentino.

En el mandato de Gustavo Bevilacqua (2011-2015), la desaparición del área específica del organigrama municipal evidencia un estancamiento en el accionar internacional basada en el sostenimiento de los acuerdos bilaterales y las estrategias multilaterales en vigencia, y el abordaje de aspectos meramente protocolares de la participación internacional.

En cuanto las estrategias implementadas se destaca su diversidad: a) hermanamientos de ciudades; registrándose ocho ciudades hermanas: en Estados Unidos, Chile, España, Italia, Cuba, Israel, Perú y China; b) membresía activa en la Red Mercociudades; c) participación del Comité de Integración de la Región de los Lagos, compuesto por otras tres provincias argentinas y 
las cuatro regiones colindantes de Chile proceso que ha impulsado el desarrollo del Corredor Bioceánico del Sur del que Bahía Blanca forma parte; d) Rondas de Negocios, Misiones Comerciales y Ferias Internacionales e) desarrollo de programas y proyectos para el posicionamiento internacional a través del proceso de construcción de la Marca de Bahía Blanca.

En relación a las temáticas abordadas y los objetivos perseguidos, la mayoría de las iniciativas apuntan a fomentar el comercio exterior, la diversificación de mercados, la asociación con actores de similares características de ciudad-puerto o tradiciones demográfico-culturales afines; la integración con provincias norpatagónicas y, por medio de ellas, con las regiones chilenas fronterizas; el desarrollo de obras de infraestructura, y además, la búsqueda de que la identidad cultural de Bahía Blanca trascienda en su rol de capital regional, a través de una estrategia general de Marca Ciudad.

En este marco cabe realzar dos espacios de actuación distintivos: la participación de la Red Mercociudades y la organización de Rondas de Negocios, Misiones Comerciales y la participación en Ferias Internacionales.

En relación a la primera, ejerce la coordinación y subcordinación de unidades temáticas. Asimismo, se destaca la correspondencia con la estrategia general de exportaciones a nivel nacional y además, que la participación en esta red y los hermanamientos fomenta la generación de políticas locales de comercio exterior.

En cuanto a la segunda, se ha visto el estrecho accionar con el Ministerio de Relaciones Exteriores y Culto, la Fundación Export.Ar, el Gobierno de la Provincia de Buenos Aires, con Intendentes y Gobernadores de otras provincias asentadas en el Corredor Bioceánico del Sur e, igualmente con otras dependencias municipales a través de capacitaciones, misiones comerciales y desarrollo de proyectos que demuestran el grado de articulación entre los niveles de gobierno.

En cuanto a los actores que participan de este relacionamiento son centrales las universidades públicas que mantienen una estrecha colaboración en las iniciativas internacionales emprendidas; los Consulados, que junto la presencia de Asociaciones y Federaciones Regionales de Inmigrantes también trabajan asociadas al municipio. Asimismo, se destaca la vinculación con el Centro de Despachantes de Aduana, Cámara de Comercio de Bahía Blanca, Corporación del Comercio, Industria y Servicios de Bahía Blanca, Polo Tecnológico Bahía Blanca, entre otras entidades.

La suma de estas cuestiones ha permitido afirmar que resulta un ejemplo 
paradigmático a nivel nacional y provincial dentro del universo de municipios intermedios dada la existencia de organismos públicos y sectores no gubernamentales y privados con una importante trayectoria en la materia. Adicionalmente, el dinamismo de dichas actividades estuvo muy ligado al perfil de liderazgo e intereses de los intendentes en gestión y a la concepción de los mismos sobre la relevancia de las acciones internacionales para alcanzar localmente sus objetivos de desarrollo.

A pesar de los avances en la vinculación con el exterior, es necesario reconocer que en la práctica, no todos los gobiernos locales pueden conducir sus propios intereses y agenda internacional a través de estrategias propias. En algunos casos, como el tratado, si bien establecen un interés por internacionalizarse e incluso un área gubernamental sobre la temática, la construcción de una política internacional requiere de una decisión política que genere en principio nuevas capacidades institucionales materiales y humanas que permitan aprovechar el entorno internacional desde una mirada estratégica.

En ese sentido, la voluntad política es imprescindible para la construcción y la implementación de una agenda internacional articulada con las demás prioridades de la gestión.

Estas consideraciones finales dejan en evidencia la necesidad de proseguir con la investigación para profundizar el análisis, se pretende haber realizado un aporte empírico a la temática a modo de detección de fortalezas y debilidades de la política internacional subnacional de un municipio intermedio argentino. 


\section{REFERENCIAS B I B L I G RÁ I C A S Y FUENTES}

Batista, S., Jakobsen, K. y Evangelista, A. (2008). La apertura al exterior de las ciudades latinoamericanas y la cooperación descentralizada. Montevideo: Observatorio de Cooperación Descentralizada Unión Europea - América Latina.

Borja J. y Castells M. (1997). Local y global. La gestión de las ciudades en la era de la información. Barcelona: UNCHS, Santillana S.S. Taurus.

Calvento, M. (2012). La participación internacional de los estados subnacionales: El caso de los gobiernos municipales de tamaño intermedio de la provincia de Buenos Aires. Tesis para alcanzar el título de Doctora en Ciencia Política, Universidad Nacional de General San Martín (UNSAM), Buenos Aires, Argentina.

Calvento, M., Herrero, M. S., \& Lorenzo, N. (2014). “Análisis teórico-empírico de la gestión internacional de los actores subnacionales: una propuesta de abordaje para las ciudades intermedias". Revista CONfines, 20(10), 35-58.

Calvento, M. (2016). “La política internacional subnacional: una propuesta para el abordaje del accionar contemporáneo en Argentina". Revista Desafíos, 28(I), 295-332. Doi: dx.doi.org/10.12804/desafios28.1.2016.07

CIPPEC (2012). La Cooperación Internacional como herramienta para el Desarrollo Local. Buenos Aires: Centro de Implementación de Políticas Públicas para la Equidad y el Crecimiento (CIPPEC).

Colacrai, M. (2010). “El tejido de Relaciones Bilaterales desde el Ámbito Subnacional. Desarrollo de una innovadora interdependencia entre la Argentina y Chile". En Maira, L. (Ed), La Política Internacional Subnacional en América Latina (pp.305-327). Buenos Aires: Libros del Zorzal.

Colacrai, M. (2013) "El rol de las ciudades en el actual escenario internacional y en los procesos de integración regional: ni tanto, ni tan poco". Revista de Integración y Cooperación Internacional, N ${ }^{\circ} 14$ CERIR, Enero/Marzo2013, 8-14. [en línea]. [consulta: 25/05/2016]. Disponible en http://www.cerir.com.ar/admin/_cerir/archivos/publicaciones/

Colacrai, M. (2016). “Cuando la frontera dialoga: Singularidades de la relación argentino-chilena en las últimas décadas". Revista Estudios Fronterizos, 17(34), 85-99, http:// dx.doi.org/10.21670/ref.2016.34.a05

Colacrai, M. y Zubelzú, G. (1998). “El creciente protagonismo externo de las provincias argentinas". En CERIR, La política exterior argentina 1994-1997 (pp. 319-322). Rosario: Ediciones CERIR.

Colacrai, M. y Zubelzú, G. (2004). “Las Vinculaciones Externas y la Capacidad de Gestión Internacional Desplegadas por las Provincias Argentinas en la Última Década. Una Lectura Desde Las Relaciones Internacionales". Buenos Aires: CARI. [en línea]. [consulta: 15/04/2016]. Disponible en: www.cari.org.ar/pdf/zubelzu-colacrai.pdf.

Consultora Pontis. (2012). Las áreas internacionales de los municipios argentinos. 
Buenos Aires. Recuperado 8 de Marzo de 2017, de http://www.pontisweb.com.

CREEBBA (2014a) Producto Bruto del Partido de Bahía Blanca. En Indicadores de Actividad Económica (IAE), No 137, año 24, Noviembre de 2014, 8-15. Recuperado 13 de Febrero de 2017, de http://www.creebba.org.ar/iae/iae137/Aporte_de_la_construccion_privada_al_producto_bruto_local_en_2013_IAE_137.pdf

CREEBBA (2015) Comercio Exterior de Bahía Blanca en 2014. En Indicadores de Actividad Económica (IAE), N ${ }^{\circ}$ 140, año 25, Diciembre de 2014, 10-14. Recuperado 12 de Febrero de 2017, de http://www.creebba.org.ar/iae/iae140/Comercio_exterior_de_Bahia_Blanca_en_2014_IAE_140.pdf.

CREEBBA. (2004), Corredor Bioceánico: Competitividad de sistemas logísticos II. En Indicadores de Actividad Económica (IAE), N 74, año 14, mayo 2004, Bahía Blanca, Argentina. Recuperado 3 de Marzo de 2017, de http://www.creebba.org.ar/iae/iae74/Competitividad_de_sistemas_logisticos_el_Corredor_Trasandino_del_Sur_(II)_IAE_74.pdf.

CREEBBA. (2011) Oportunidades de intercambio en el Corredor Trasandino del Sur. En Indicadores de Actividad Económica (IAE), $\mathrm{N}^{\circ}$ 116, año 21, mayo 2011, 15-22. Recuperado 31 de Marzo de 2017, de http://www.creebba.org.ar/iae/iae116/ Oportunidades_de_ intercambio_en_el_Corredor_Trasandino del Sur IAE_116.pdf.

Dabat, G. (2004). Politicas Locales de Comercio Exterior en la Argentina: Comercio y desarrollo desde una perspectiva endogenista. Quilmes: Universidad Nacional de Quilmes. [en línea]. [consulta: 20/03/2016]. Disponible en: http://ici.unq.edu.ar/ici_biblio.html.

De Marsilio, E. G. (2006). Aportes Teórico - Metodológicos para el estudio de las Relaciones Paradiplomáticas. Qué, Quiénes, y Cómo. Análisis Del Caso Argentino. Serie Breviario en Relaciones Internacionales. Córdoba, Argentina.

Di Meglio F. y Nicolao, J. (2016). “La política internacional subnacional del Municipio de Bahía Blanca. Un análisis de sus capacidades estatales (2011-2015)”. En Mariana

Calvento (Comp.) Gestión y Política Internacional Subnacional. El caso de los Municipios del Interior de la Provincia de Buenos Aires, (pp.99-122). Tandil: CEIPIL-ANPCyT.

Duchacek, I. (1986). Las dimensiones territoriales de las politicas: Dentro, entre y a través de las Naciones. Boulder Colorado: Westview Press.

Forni, P. (2009). Triangulación. Madrid: Mimeo.

García Segura, C. (1996). “La Actividad Exterior de las Entidades Políticas Subestatales". Revista de Estudios Políticos (Nueva Época), No 91, Enero-Marzo 1996, 235-264.

Gorenstein, S. (2004). “De la convertibilidad a la post-devaluación: impactos y nuevas tendencias económicas en la ciudad-región de Bahía Blanca". Ponencia presentada en las Segundas Jornadas de Historia Regional Comparada y Primeras Jornadas de Economía Regional Comparada, 3-6 de octubre de 2004 Porto Alegre, Pontificia Universidad Católica de Rio Grande do Sul. . Recuperado 8 de Marzo de 2017 de http://www.fee. tche.br/sitefee/download/jornadas/2/e1-04.pdf 
Gorenstein, S. (2004). Perfiles Económicos de Bahía Blanca y la Región: Entre el Complejo Agroexportador y el Complejo Petroquímico. En Plan Fénix Propuesta para el desarrollo con equidad: Economías Regionales - Segundo Encuentro de Universidades Nacionales, Plan Fénix, (pp. 201-218). Buenos Aires: Universidad de Buenos Aires - Universidad de Río Cuarto.

Hernández Sampieri, R., Fernández, C. y Baptista, P. (2006). Metodología de la investigación 4ed. México D. F.: McGraw-Hill Interamericana.

Herrero, M. S. (2016). La actuación internacional de los estados subnacionales y la federalización de la política exterior argentina: el caso del Municipio de Bahía Blanca. Tesis para alcanzar el título de Magister en Cs. Sociales, UNICEN. Tandil, Argentina.

Herrero, M.S. (2014). La inserción internacional de los estados subnacionales: Bahía Blanca como caso de estudio. Tesis para alcanzar el título de Licenciada. Licenciatura en Relaciones Internacionales, UNICEN. Tandil. Argentina.

Maxwell, J. A. (1992). “Understanding validity in qualitative research". Harvard Educational Review 62 (3): 279-300.

Michelini, J.J. y Davies, C. (2009). Ciudades intermedias y desarrollo territorial: un análisis exploratorio del caso argentino. Documento de Trabajo N5. Madrid: GEDESUR.

Müller, P. (2006). Las políticas públicas. Universidad Externado de Colombia: Buenos Aires.

Nicolao, Julieta (2015) “Representaciones extranjeras, desarrollo local y gestión internacional de los gobiernos subnacionales. El rol de los consulados y las organizaciones de migrantes". En M. Calvento (Comp.), Procesos y actores en la Gestión de la Política internacional Subnacional. (pp. 113-140). Tandil: CEIPIL.

Observatorio PyME Regional Suroeste de la Provincia de Buenos Aires. (2008). Industria Manufacturera. Año 2007. Buenos Aires: Fundación Ob $\neg$ servatorio PyME, Universidad Tecnológica Nacional, Bononiae Libris.

Paquin, S. (2004). Paradiplomatie et Relations Internationales: Théorie des Straté- gies Internationales des Régions Face à la Mondialisation. Bruselas: Presses Interuniversitaires Européennes.

Ponce Adame, E. A. (2011). “Historia y actualidad de la acción exterior de los gobiernos locales". Revista Trabajos de Investigación en Paradiplomacia, 1, 10-43.

Quartucci, Elisa (2013) El posicionamiento competitivo de las ciudades en el sistema nacional e internacional. El caso del gobierno de la ciudad de Bahía Blanca. Tesis para alcanzar el título Magister en Políticas y Estrategias, Universidad Nacional del Sur, Bahia Blanca, Argentina.

Reyes, P. y Hernández, A. (2008). “El estudio de caso en el contexto de la crisis de la modernidad". Cinta de Moebio 32: 70-89. [en línea]. [consulta: 15 /06/2016]. Disponible en: www.moebio.uchile.cl/32/reyes.html 
Russell, R. (2010). “El Estado- Nación y los actores gubernamentales no centrales: una relación complementaria". En Maira, L. (Editor), La Política Internacional Subnacional en América Latina (pp. 83-106). Buenos Aires: Libros del Zorzal.

Salomón, M. (2007). La Acción Exterior de los Gobiernos Subnacionales y el Análisis de Políticas Exteriores. Brasilia: 1er Encontro Nacional da ABRI Política Externa.

Soldatos, P. (1990). "An Explanatory Framework for the Study of Federated States as Foreign-policy Actors". En Michelman, H.J. y Soldatos, P (Eds.) Federalism and International Relations. The Role of Subnational Units, (pp.34-58). Oxford: Clarendon Press.

Todd, Z., y Nerlich, B. (2004). "Future directions". En Todd Z., Nerlich B., McKeown B. y Clarke D. (Eds.), Mixing methods in psychology (pp. 231-237). Berlin/New York: Mouton de Gruyter.

Vapñarski, C. y Gorojsvsky, N. (1990). El crecimiento urbano en la Argentina. Buenos Aires: GEL.

Vigevani, T. (2006). "Problemas para a atividade internacional das unidades subnacionais. Estados e municípios brasileiros", Revista Brasileira de Ciências Sociais, 21(62).

\section{F UENTES}

-Dirección Provincial de Estadística de la Provincia de Buenos Aires. Ministerio de Economía. (2005): Producto Bruto Geográfico, Desagregación Municipal año 1993 Recuperado 8 de Marzo de 2017, de http://www.ec.gba.gov.ar/Estadistica/pdf/PresentacionPBG.pdf.

-INDEC (2010). Censo Nacional de Población, Hogares y Vivienda, 2001. [en línea]. consulta: [6/6/2014]. Disponible en: http://www.indec.gov.ar.

-Poder Ejecutivo Local. Decreto 110 Nº 1.808 /2008

-Poder Legislativo Local .Ordenanza Concejo Deliberante Bahía Blanca No 7.453/ 2003

-Poder Legislativo Local. Ordenanza Concejo Deliberante Bahía Blanca Nº 14.494/2007

-Poder Legislativo Local .Ordenanza Concejo Deliberante No 14969/2008, Decreto de Promulgación del Intendente Municipal Nº 1946/2008

-Poder Legislativo Local. Ordenanza Concejo Deliberante Bahía Blanca Nº 14.640/ 2008 


\section{NFORMESTÉCNICOS Y DE GESTIÓN \\ DE ORGA NISMOS NACIONALESE \\ I N T E R N A I O N A L E S}

- Cámara de Empresas de Software y Servicios Informáticos de Argentina (CESSI) (2015) Memoria 2015, Buenos Aires, Argentina.

- Consorcio de Gestión del Puerto de Bahía Blanca (2015) Estadísticas. Destinos de las Exportaciones, 2015.

- MERCOCIUDADES (2010). 15 años de Mercociudades. Rosario: Municipalidad de Rosario Secretaría Ejecutiva Mercociudades

- MERCOCIUDADES (2011). Boletín Electrónico de la Unidad Temática de Desarrollo Económico Local - Red Mercociudades. Número 05/ 2011. Guarulhos: Mercociudades

- Municipalidad de Bahía Blanca Secretaria de Ceremonial y Protocolo. (2005). Informe de Gestión. Bahía Blanca: Municipalidad de Bahía Blanca.

- Municipalidad de Bahía Blanca. (2009). Informe de Gestión 2008-2009. Plan de Gestión Municipal. La ciudad del Bicentenario. Bahía Blanca 2008-2010. Bahía Blanca: Municipalidad de Bahía Blanca.

- Municipalidad de Bahía Blanca. (2011). Informe del Área de Relaciones Internacionales. Dirección de Asuntos Turísticos e Internacionales. Bahía Blanca: Municipalidad de Bahía Blanca.

\section{I S C URSOS E I N T E RVE N C I O N S P ÚB L I A S DE FUNCIONARIOS}

Breitenstein, Cristian (2007) Discurso de Asunción ante el Concejo Deliberante. Bahía Blanca, 10 de Diciembre de 2007.

\section{P R E N S A}

Breitenstein C. (2011) Marca ciudad. [en línea]. Recuperado 10 de Diciembre de 2016 de http:// bahiablanca-gestionmunicipal.blogspot.com.ar/2011/07/marca-ciudad. html\#!/2011/07/marca-ciudad.htm. 
